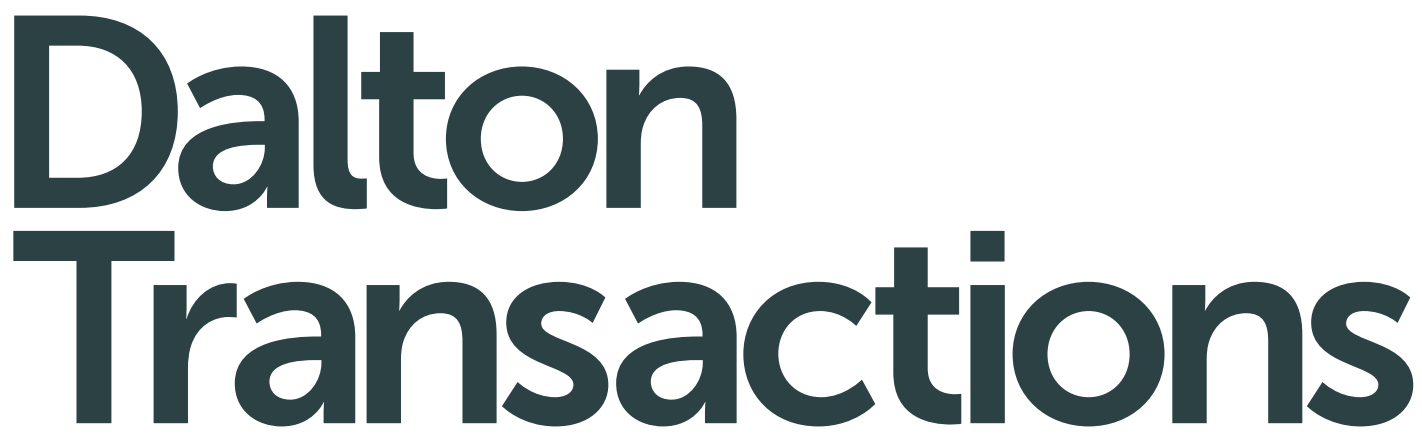

An international journal of inorganic chemistry

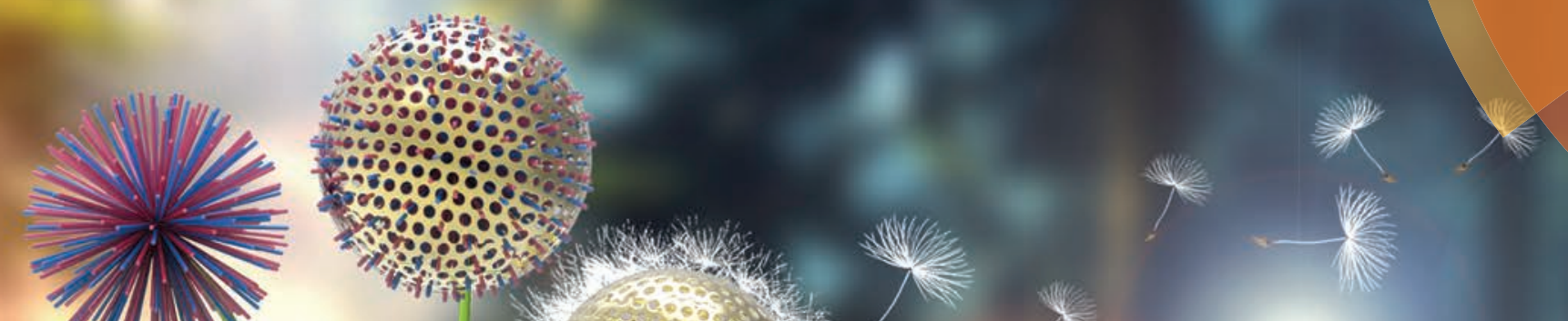

ISSN 1477-9226

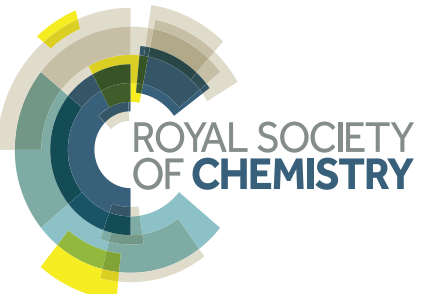

\section{PAPER}

Fangyuan Gai, Farid Akhtar et al.

Mixed anionic surfactant-templated mesoporous silica nanoparticles for fluorescence detection of $\mathrm{Fe}^{3+}$
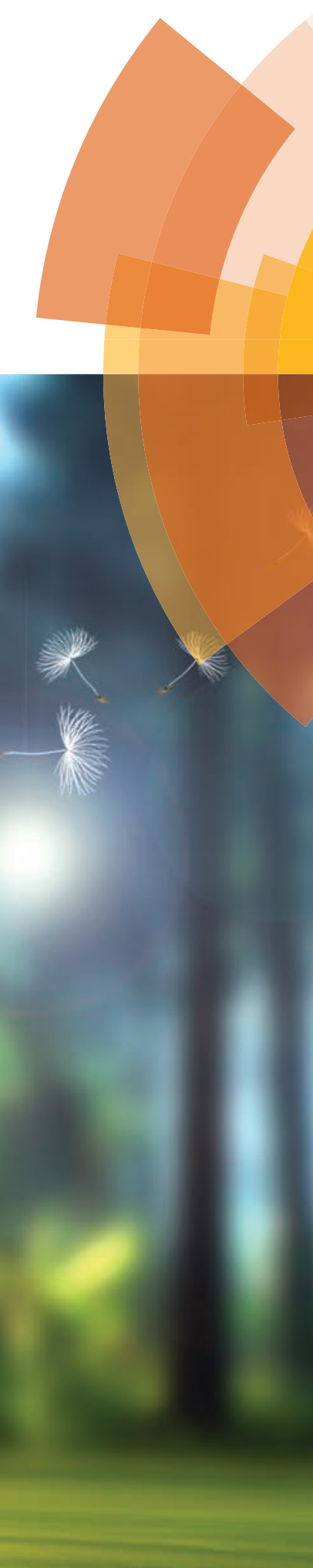


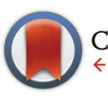

CrossMark \& click for updates

Cite this: Dalton Trans., 2016, 45 508

Received 7th August 2015,

Accepted 14th November 2015

DOI: $10.1039 / \mathrm{c} 5 \mathrm{dt}$ t03052h

www.rsc.org/dalton

\title{
Mixed anionic surfactant-templated mesoporous silica nanoparticles for fluorescence detection of $\mathrm{Fe}^{3+}+$
}

\author{
Fangyuan Gai, ${ }^{a}$ a,b,c Tianlei Zhou, ${ }^{d}$ Guang Chu, ${ }^{c}$ Ye Li, ${ }^{e}$ Yunling Liu, ${ }^{c}$ Qisheng Huo ${ }^{c}$ \\ and Farid Akhtar*b
}

\begin{abstract}
This work demonstrates a novel method for the synthesis of large pore mesoporous silica nanoparticles (MSNs) with a pore diameter of $10.3 \mathrm{~nm}$ and a particle diameter of $\sim 50 \mathrm{~nm}$ based on the incorporation of mixed anionic surfactants sodium dodecyl benzene sulfonate (SDBS) and sodium dodecyl sulphate (SDS) as the template in the synthesis process. The dispersity, morphology, pore structure and size of mesoporous nanoparticles were adjusted by changing the molar ratio of two anionic surfactants, the concentration of the co-structure-directing agent (3-aminopropyltrimethoxysilane) and the reaction temperature. The results of synthesis experiments suggested that the formation of large pore MSNs involved a nucleation and growth process. MSNs were post-grafted with a Schiff base moiety for fluorescence sensing of $\mathrm{Fe}^{3+}$ in water. The applicability of functionalized MSNs was demonstrated by selective fluorescence detection of $\mathrm{Fe}^{3+}$ in aqueous media.
\end{abstract}

\section{Introduction}

Mesoporous silica nanoparticles (MSNs) have attracted increasing attention because of their unique and tuneable pore structures, which potentially could be applied as drug carriers, catalyst supports and adsorbents in biomedical and other fields. ${ }^{1,2}$ Various templating approaches have been developed in the past few decades to synthesize mesoporous silica nanoparticles with well-defined topology and morphology using different surfactants as soft-templates. ${ }^{3-7}$ For example, Zhao and co-workers synthesized biodegradable MSNs with a tuneable pore size by using cetyltrimethylammonium chloride (CTAC) as a cationic surfactant template. ${ }^{8}$ Mou and co-workers designed and synthesized a series of cationic surfactanttemplated MSNs for intracellular drug delivery and bio-

\footnotetext{
${ }^{a}$ Department of Petroleum and Chemical Engineering, Dalian University of Technology, 2 Dagong Road, New District of Liaodong Bay, Panjin 124221, China. E-mail: gaifangyuan@dlut.edu.cn

${ }^{b}$ Division of Materials Science, Luleå University of Technology, SE-97187 Luleå, Sweden

${ }^{c}$ State Key Laboratory of Inorganic Synthesis and Preparative Chemistry, College of Chemistry, Jilin University, Changchun 130012, China. Tel: $+86-431-85168602$

${ }^{d}$ Chemical \& Materials Engineering Department, University of Nevada at Reno, Reno, NV 89557, USA

${ }^{e}$ Institute of Chemical Materials, China Academy of Engineering Physics, Mianyang, Sichuan 621900, People's Republic of China

$\dagger$ Electronic supplementary information (ESI) available: The TEM image of MSN-4 synthesized and calcined at different reactant concentrations and temperatures. See DOI: $10.1039 / \mathrm{c} 5 \mathrm{dt} 03052 \mathrm{~h}$
}

imaging. ${ }^{9-11} \mathrm{Yu}$ and co-workers presented the synthesis of silica ellipsoids with a hexagonal mesostructure via an organic-inorganic cooperative assembly process by using the amphiphilic copolymer P123 as a template. ${ }^{12}$ Che and coworkers introduced co-structure directing agents to synthesize anionic surfactant-templated silica nanoparticles with highly ordered structures. ${ }^{13-15}$ Besides these, design and synthesis of surfactants, ${ }^{3,16-21}$ different pore structures ${ }^{22-26}$ and MSN morphologies ${ }^{27-30}$ have been proposed.

Particularly, it is important to synthesize MSNs with large mesopores, which offer superior mass transfer characteristics for nucleic acids and gene delivery for medical applications. ${ }^{31}$ There are two major pathways to synthesize large pore MSNs. One is to expand the mesopores with swelling agents such as toluene, 1,3,5-trimethylbenzene (TMB) and octanoic acid. ${ }^{32-35}$ Second is to utilize nonionic surfactants or surfactants with long hydrophobic chains as soft templates. ${ }^{36,37}$ For instance, a series of large-pore ordered mesoporous materials have been prepared by using a surfactant paired with a micelle swelling agent. Single-micelle-templated organosilica and silica nanospheres can reach large interiors (up to $\sim 18.5 \mathrm{~nm}$ in diameter) using this approach. ${ }^{21,38-40}$

Although cationic and amphiphilic surfactants are typically used to synthesize large pore MSNs, ${ }^{31}$ reports on the use of anionic surfactants to synthesize large pore MSNs are scarce. Chen and co-workers prepared core-shell hollow mesoporous nanoparticles with amino-functionalized channels by using $N$-lauroylsarcosine sodium as an anionic surfactant. ${ }^{41}$ The pore diameters of the core-shell MSNs were about $3 \mathrm{~nm}$. 
Che and co-workers designed and synthesized a series of anionic surfactant-templated nanoparticles by introducing a co-structured directing agent. ${ }^{13,15,16}$ In their work, ordered mesoporous and chiral mesoporous silica was prepared and anionic surfactant-templated mesoporous silica displayed a highly ordered 2D-hexagonal $\mathrm{p} 6 \mathrm{~mm}$ mesoporous structure with the pore diameter tuned to $6.2 \mathrm{~nm}$. Despite the progress, it is still a challenge today to prepare larger pore MSNs by using anionic surfactants without a swelling agent or polymer micelle.

Anionic surfactants such as alkyl carboxylic acids, phosphoric acid, sulfonic acid and their corresponding sodium salts are consumed in a variety of fields because of their low cost, environment friendliness and low toxicity. ${ }^{13}$ In addition, the feasibility of using different surfactants or catalysts has been explored; ${ }^{42}$ few effects of using mixed short carbon chained surfactants have been studied to synthesize large pore MSNs. ${ }^{43}$ By adjusting the ratio of mixed cationic surfactants and the temperature of reaction, Qiao and co-workers proposed and discussed stellate mesopore nanoparticles with large pores for intracellular drug delivery. ${ }^{43}$

In this study, by using mixed anionic surfactants sodium dodecyl benzene sulfonate (SDBS) and sodium dodecyl sulphate (SDS) as the template, large pore MSNs have been synthesized without using a swelling agent or polymer micelle. The morphology, structure and monodispersity of nanoparticles were tuned by adjusting the concentration of SDBS and APTS (3-aminopropyltrimethoxysilane). The nucleationgrowth process has been proposed and discussed to be involved in the formation of mesoporous silica nanoparticles. The diameter of mesopores of silica nanoparticles was measured by transmission electron microscopy (TEM) and $\mathrm{N}_{2}$ adsorption analysis after the extraction and calcination processes. Because the MSNs are functionalized with the amino group, various proteins and organic moieties can be postgrafted on the surface of MSNs. We have grafted nanoparticles with a Schiff base and reported $\mathrm{Fe}^{3+}$ fluorescence sensing in aqueous media.

\section{Experimental section}

\section{Materials}

Tetraethylorthosilicate (TEOS) and (3-aminopropyl)-triethoxysilane (APTS) were purchased from Sigma-Aldrich. Sodium dodecyl benzene sulfonate (SDBS) and sodium dodecyl sulphate (SDS) were purchased from Aladdin Industrial Inc. Acetic acid $\left(\mathrm{CH}_{3} \mathrm{COOH}, \geq 99.5 \%\right)$, 4-benzhydrylbenzaldehyde (TPA), hydrochloric acid ( $\mathrm{HCl}$, fuming, $\geq 37 \%$ ), and anhydrous ferric chloride $\left(\mathrm{FeCl}_{3}, \geq 97 \%\right)$ were purchased from Alfa Aesar. All reagents and solvents were used without further purification. Deionised water was used in the experiment (resistivity $\geq 18.2 \mathrm{M} \Omega$ ).

\section{Synthesis of MSNs}

MSNs were synthesized using TEOS and APTS as the silica precursors, hydrochloric acid ( $\mathrm{HCl}$, fuming, $\geq 37 \%$ ) as the acidic
Table 1 Synthesis conditions of different mesoporous silica nanoparticles (MSNs)

\begin{tabular}{llllllll}
\hline & $\begin{array}{l}\text { SDS } \\
(\mathrm{g})\end{array}$ & $\begin{array}{l}\text { SDBS } \\
(\mathrm{g})\end{array}$ & $\begin{array}{l}\mathrm{H}_{2} \mathrm{O} \\
(\mathrm{mL})\end{array}$ & $\begin{array}{l}\mathrm{HCl} \\
(\mathrm{g})\end{array}$ & $\begin{array}{l}\text { TEOS } \\
(\mathrm{mL})\end{array}$ & $\begin{array}{l}\text { APTS } \\
(\mathrm{mL})\end{array}$ & $\begin{array}{l}T \\
\left({ }^{\circ} \mathrm{C}\right)\end{array}$ \\
\hline MSN-1 & 0.072 & 0 & 8.75 & 0.5 & 0.375 & 0.020 & 40 \\
MSN-2 & 0.072 & 0 & 8.75 & 0.5 & 0.375 & 0.040 & 40 \\
MSN-3 & 0.060 & 0.014 & 8.75 & 0.5 & 0.375 & 0.030 & 40 \\
MSN-4 & 0.060 & 0.014 & 8.75 & 0.5 & 0.375 & 0.040 & 40 \\
MSN-5 & 0.060 & 0.014 & 8.75 & 0.5 & 0.375 & 0.040 & 25 \\
& & & & & & & \\
\hline
\end{tabular}

catalyst, and SDBS and/or SDS as the anionic surfactants. In fact, APTS not only worked as the co-condensation silica precursor to introduce the amino group, but also as the costructure directing agent (CSDA) to form the morphology of nanoparticles and mesopores. All types of MSNs were formed under acidic conditions; the synthesis system contained $0.1 \mathrm{M}$ HCl. By tuning the concentration of SDBS and APTES and the temperature of the synthesis processes, products with different shapes and dispersities have been observed by TEM. The synthesis conditions and the physical parameters of samples are summarized respectively in Table 1.

In a typical synthesis of MSNs, a given amount of SDS $(0.06 \mathrm{~g}) / \mathrm{SDBS}(0.014 \mathrm{~g})$ and $\mathrm{HCl}(0.1 \mathrm{M}, 0.5 \mathrm{~g})$ was first dissolved in $8.75 \mathrm{~mL}$ DI water to form a clear micelle solution at a reaction temperature of $40{ }^{\circ} \mathrm{C}$. After stirring the solution for $0.5 \mathrm{~h}, 0.375 \mathrm{~mL}$ of TEOS and $0.04 \mathrm{~mL}$ of APTS were quickly added to the solution. After an additional $4 \mathrm{~h}$ of stirring, the as-synthesized products were separated by centrifugation at $10000 \mathrm{rpm}$ for $8 \mathrm{~min}$ or filtration. The separated white powder was washed with DI water and ethanol to remove the surfactant on the surface of MSNs. After that, MSNs were dried in an oven at $60{ }^{\circ} \mathrm{C}$ for $24 \mathrm{~h}$.

The anionic surfactant templates were removed by both extraction from reflux and washing with $\mathrm{EtOH}$ and $\mathrm{HCl}$ with a molar ratio of $1: 1$ and calcination at $600{ }^{\circ} \mathrm{C}$ for $5 \mathrm{~h}$ with a ramping rate of $1^{\circ} \mathrm{C} \mathrm{min}^{-1}$ in air.

\section{Characterization}

Transmission electron microscopy (TEM) was carried out using an FEI Tecnai G2 F20 s-twin D573 operated at $200 \mathrm{kV}$. The specimens for TEM were prepared by directly placing a small drop of the nanoparticle suspension on a carbon-coated TEM grid. Hitachi S-4800 field-emission scanning electron microscopy (FE-SEM) has been used in the test of morphologies. The IR spectra were obtained with a Bruker IFS 66v/ S FTIR spectrometer. The adsorption-desorption isotherms of nitrogen were obtained at $77 \mathrm{~K}$ using a Micromeritics TriStar 3000 system. Adsorption isotherms of $\mathrm{N}_{2}$ at $77 \mathrm{~K}$ were used to calculate the pore size distributions based on the BJH model. The fluorescence emission spectra of the samples were recorded on a HORIBA Instrument FluoroMax - Compact spectrofluorometer. 


\section{Results and discussion}

In this study, our basic idea is to prepare large pore MSNs by using mixed anionic surfactants as the template. By changing the concentration of SDBS and co-structure directing agent (APTS) and the reaction temperature, we investigated the formation process of large pore MSNs. After analysis of the relationship between the morphology and dispersity under different experimental conditions (summarized in Table 1), a nucleation-growth process for the MSN formation has been elucidated. Finally, MSN-4 was post-grafted with triphenylamine and Schiff base moieties, which demonstrated good sensing ability towards $\mathrm{Fe}^{3+}$.

\section{Effect of mixed anionic surfactant and APTS}

The morphology characterization by TEM (Fig. 1) of the synthesized nanoparticles suggests that without SDBS, mesoporous silica nanoparticles can be synthesized using SDS and APTS (Fig. 1a, e, b and f). When the concentration of SDS was $0.72 \mathrm{~g} \mathrm{~mL}^{-1}$ and the amount of APTS was $0.02 \mathrm{~mL}$, the as- synthesized MSN-1 displayed aggregated morphology and the synthesis product contained a large fraction of mesoporous silica monoliths with a pore diameter of 5-6 nm approximately measured from the TEM image. MSN-1 were nonuniform in size from $40 \mathrm{~nm}$ to $100 \mathrm{~nm}$ and showed different particle morphologies (Fig. 1a and e). On further addition of APTS (0.03 or $0.04 \mathrm{~mL}$ ) into the synthesis system, as shown in Fig. $1 \mathrm{~b}$ and f, the dispersity of nanoparticles (MSN-2) improved. However, MSN-2 showed different particle morphologies and pore structures. In addition, we found that MSN-2 were frangible. The fragmented particles of MSN-2 are shown in Fig. 1b and f. Similar results and morphologies of MSN-1 and MSN-2 have been observed in SEM images (Fig. 2). In Fig. 2a, MSN-1 show aggregation, spherical shape and smooth surface, while MSN-2 show broken spheres (Fig. 2c). The dispersity and the uniformity of SDS-templated mesoporous silica nanoparticles were further tuned by adding an increasing amount of APTS as the co-structure directing agent (CSDA).

The synthesis was performed by using SDS alone without SDBS and APTS to evaluate the surfactant effect of SDS and the
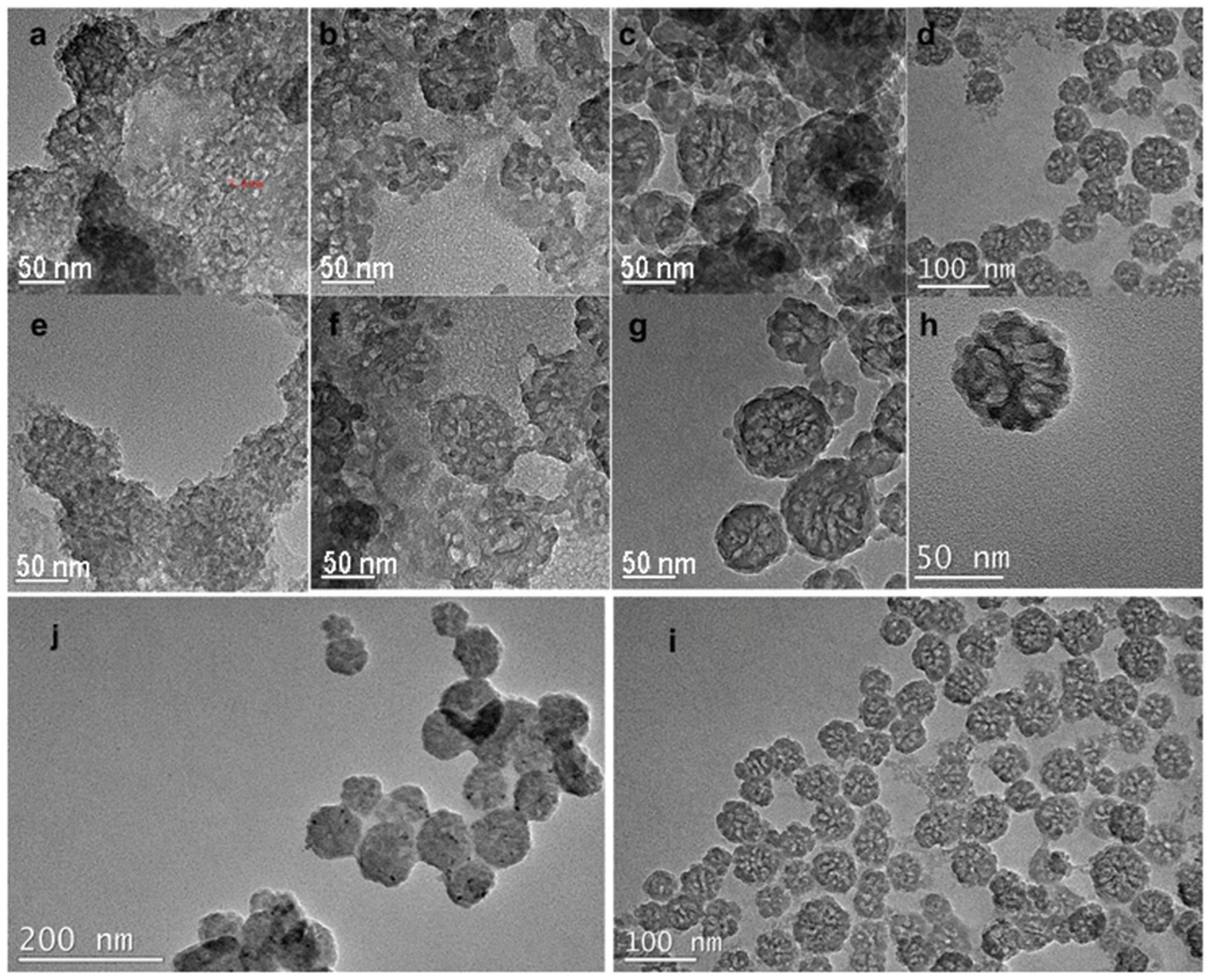

Fig. 1 TEM images of MSNs synthesized with different surfactant molar ratios of SDS and SDBS: (a) and (e) MSN-1, (b) and (f) MSN-2, (c) and (g) MSN-3, (d) and (h) MSN-4, (j) MSN-5, and (i) MSN-4. Scale bars are shown in each photo. 


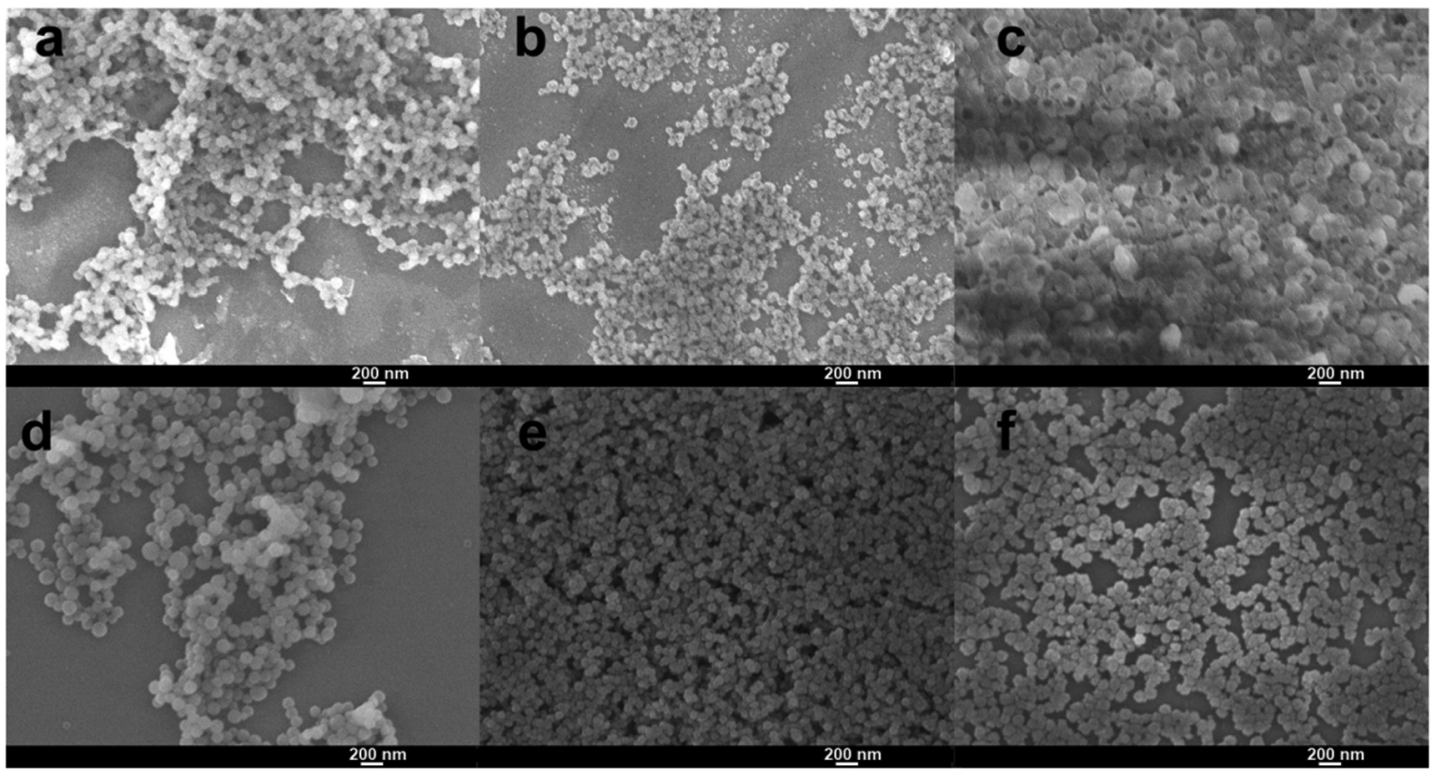

Fig. 2 SEM images of MSNs synthesized with different surfactant ratios of SDS and SDBS: (a) MSN-1, (b) and (c) MSN-2, (d) MSN-3, (e) and (f) MSN-4.

co-structure-directing effect of APTS. Large vesicles and their aggregates were formed, as shown in the TEM images in Fig. S2(a) and (b). $\dagger$ The images indicate that without the mixed surfactant and APTS, the formation of mesopores could not be realized. The same synthesis experiment was performed by using SDBS without SDS and APTS. The TEM images (Fig. S2(c) and (d) †) showed the formation of a silica gel block without mesopores. These syntheses suggest that mesopores could not form by using SDS or SDBS alone. In other words, mixed anionic surfactants (SDS and SDBS) played an important part in the formation of mesopores via a CSDA method.

To construct more stable MSNs, we used SDBS mixed with SDS as the mixed anionic surfactant template. We tried to introduce SDBS for the morphology and pore structure control of large pore MSNs. As shown in Fig. 1c and g, MSN-3 showed obvious improved dispersity compared to MSN-2 (Fig. 1b and f). The improved dispersity is attributed to the SDS replaced by SDBS in the mixed surfactant-templated synthesis system; the amount of free anions in the reaction process would become lower due to the stronger binding of tosylate over SDS. ${ }^{42,43}$ The tosylate moieties could be used to form large mesopores by an anion competition process, as discussed before, ${ }^{42}$ where the tosylate anions compete with silicate oligomers during the particle formation. ${ }^{42}$ From the observation of the SEM image of MSN-3 (Fig. 2d), we find that the nanoparticle size is not uniform. Similar results could be found in Fig. 1c and g, which show strong aggregation between particles. The particle size of MSN-3 (Fig. 1c and g) ranges from $30 \mathrm{~nm}$ to $100 \mathrm{~nm}$ with larger aggregates. However, by adding an increasing amount of APTS $(0.04 \mathrm{~g})$, uniform and monodisperse MSN-4 formed with similar pore structures to MSN-3 (Fig. 1d, h and $2 \mathrm{e}, \mathrm{f})$. Recently, the tosylate effects have been proposed by
Zhang $^{42}$ and Qiao $^{43}$ in the cationic surfactant-templated method, respectively.

From comparison of MSN-2 and MSN-4, we could find that by adding SDBS to form a mixed surfactant template, the morphology and the pore structure considerably changed (Fig. 1). We could deduce that SDBS not only contributed to the formation of the mixed template but also played an important role in controlling the pore structure, particle size and morphology. The mixed surfactant template method proposed the significance of SDBS in the formation of mesoporous silica nanoparticles with large pores, small size and good dispersity, all these kinds of properties are different from the previous work. ${ }^{44}$ Moreover, the SDS and SDBS are commercially used as short carbon chain surfactants, which have lower cost than the synthesized anionic surfactants. In comparison with a previous strategy to synthesize large pore MSNs, ${ }^{31,44}$ our method is superior to avoid the usage of swelling agents and hydrophobic polymer micelles with long carbon chains.

\section{Effect of reaction temperature}

We compared the synthesis product for the same molar ratio of reactants at $40{ }^{\circ} \mathrm{C}(\mathrm{MSN}-4)$ and $25{ }^{\circ} \mathrm{C}(\mathrm{MSN}-5)$, Table 1 . The synthesis reaction at $25{ }^{\circ} \mathrm{C}(\mathrm{MSN}-5)$ resulted in particle aggregation, different particle morphologies and pore structures of the product (Fig. 1j and i). While the TEM image (Fig. 1i) of the reaction product at $40{ }^{\circ} \mathrm{C}(\mathrm{MSN}-4)$ displayed better dispersity and uniform morphology of nanoparticles. It is convincing to state that a higher reaction temperature causes a higher rate of reaction during the synthesis process. ${ }^{43}$ Therefore, we deduce that a higher temperature would result in a higher rate of the assembly of silica precursor and mixed surfactant micelles to form large mesopores. 


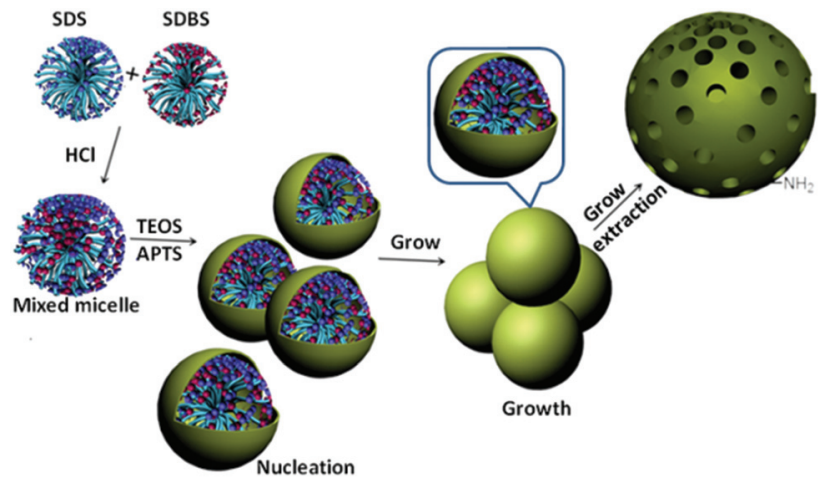

Scheme 1 Synthesis and growth route of MSN-4.

The temperature optimization results suggest that reaction kinetics plays an important role in the formation of large mesopores (Scheme 1). Compared with MSN-4, MSN-3 displayed a non-uniform size when smaller amounts of APTS were used in the synthesis process. In addition, we found that the nanoparticles had grown bigger by changing the stirring rate and the reactant concentration, as shown in Fig. S1. $\dagger$ These results are consistent with a nucleation and growth process similar to the growth process proposed by Zhang and co-workers. ${ }^{43}$

\section{$\mathrm{N}_{2}$ adsorption of $\mathrm{MSN}-4$}

By adjusting the molar ratio of SDBS in the mixed surfactant and the amount of APTS in the synthesis system, we achieved appropriate synthesis conditions for MSN-4 with large mesopores. The $\mathrm{N}_{2}$ adsorption and desorption isotherms of MSN-4 in Fig. 3a show that there are two hysteresis loops at a relative pressure of $0.6-0.8$ and $>0.9$. The hysteresis loop at $P / P_{0}>0.9$ suggests the existence of textural interparticle pores, while the hysteresis loop at $P / P_{0}$ from 0.6 to 0.8 is attributable to the framework porosity of MSN-4. The pore size distribution in Fig. 3b shows a narrow peak located at $10.3 \mathrm{~nm}$ and a broad peak at $30.7 \mathrm{~nm}$. The broad peak may be related to the textural interparticle mesopores, which is in accord with the anion competition process. ${ }^{42}$ The pore size of MSN-4 is larger than that of the mesoporous silica nanoparticles previously reported by only using one anionic surfactant as the template, ${ }^{15}$ suggesting that the mixed anionic surfactant has played an important role in the formation of large pores.

The BET surface area of MSN-4 is $217 \mathrm{~m}^{2} \mathrm{~g}^{-1}$. The surface area is similar to a previous report on mesoporous nanoparticles $\left(245 \mathrm{~m}^{2} \mathrm{~g}^{-1}\right.$ ) synthesized by using the mixed surfactant template. ${ }^{43}$ Moreover, the BET surface area is in accord with surfactant-templated large mesoporous silica reported previously. ${ }^{31,45-47}$ We measured the pore size of MSNs in the TEM image and found that the pore diameter of MSN-4 is larger than the pore diameter of MSN-1. To specifically illustrate the pore size of $\mathrm{MSN}-4$, we have marked the pore with a red line in the TEM image (Fig. S3†).
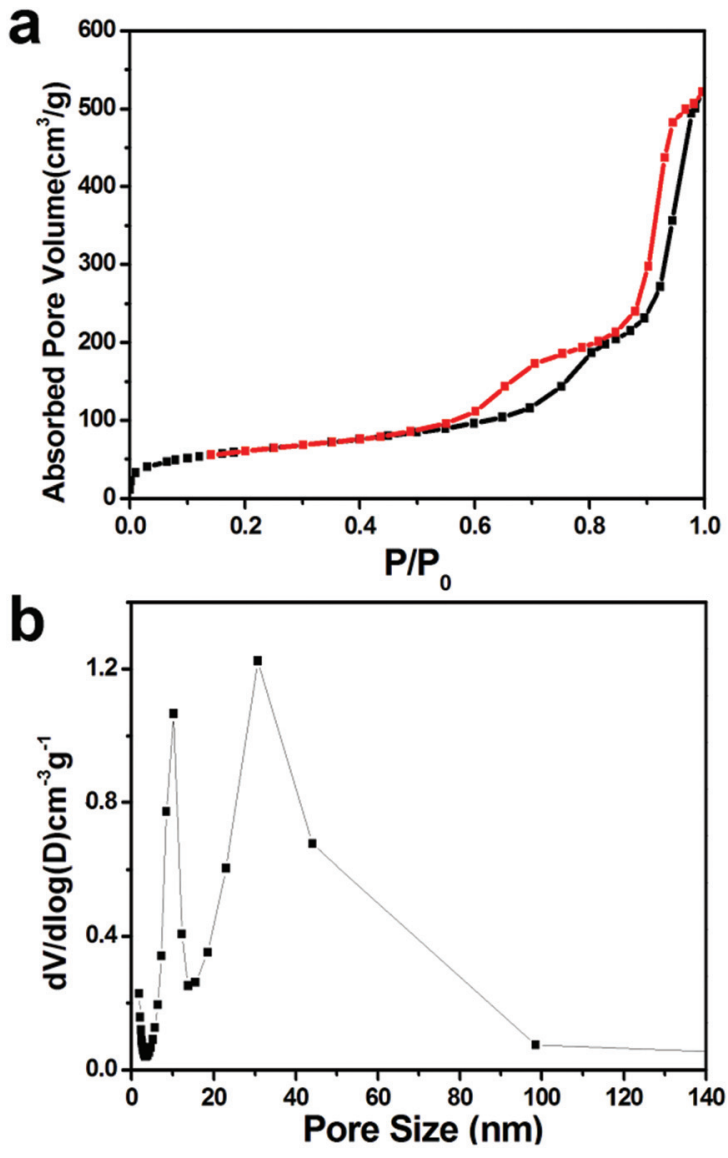

Fig. 3 Nitrogen adsorption-desorption isotherms (a) and respective pore size distributions (b) of MSN-4.

\section{Schiff based-TPA functionalized MSN-4 and $\mathrm{Fe}^{3+}$ fluorescence sensing}

To functionalize MSN-4 with Schiff based fluorescent moieties (Fig. 4a), we choose blue light emitting TPA moieties as the organic group to conjugate with the surface amino group on MSN-4 under acidic conditions. The white MSN-4 powder was dispersed in EtOH, after reflux for 3 hours under acid conditions with TPA, centrifugation has been proposed to separate Schiff based-TPA functionalized MSN-4 with a liquid. The Schiff based-TPA functionalized MSN-4 displayed yellow color, depicting a successful conjugated bonding between TPA and MSN-4. The FT-IR spectrum in Fig. 4b is in accordance with the conjugated bonding product. As shown in Fig. $4 \mathrm{~b}$, the FT-IR spectrum of the as-synthesized Schiff based-TPA functionalized MSN-4 displays peaks corresponding to silica around $790 \mathrm{~cm}^{-1}, 956 \mathrm{~cm}^{-1}$ and $1040 \mathrm{~cm}^{-1}$, which are attributed to the symmetric $\mathrm{Si}-\mathrm{O}, \mathrm{Si}-\mathrm{OH}$ and asymmetric $\mathrm{Si}-\mathrm{O}-\mathrm{Si}$ vibrations. The characteristic band of $\mathrm{C}=\mathrm{N}$ occurs around 1630-1690 $\mathrm{cm}^{-1}$, which is in good agreement with the conjugated bond between TPA and MSN-4. All the data above suggest that the Schiff base bond has been successfully formed, which gives Schiff based-TPA functionalized MSN-4. 
a

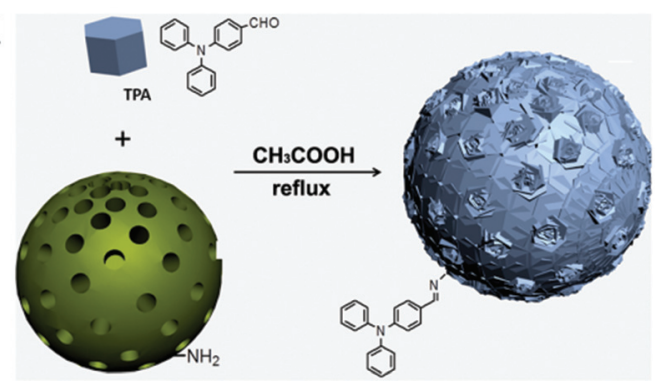

b

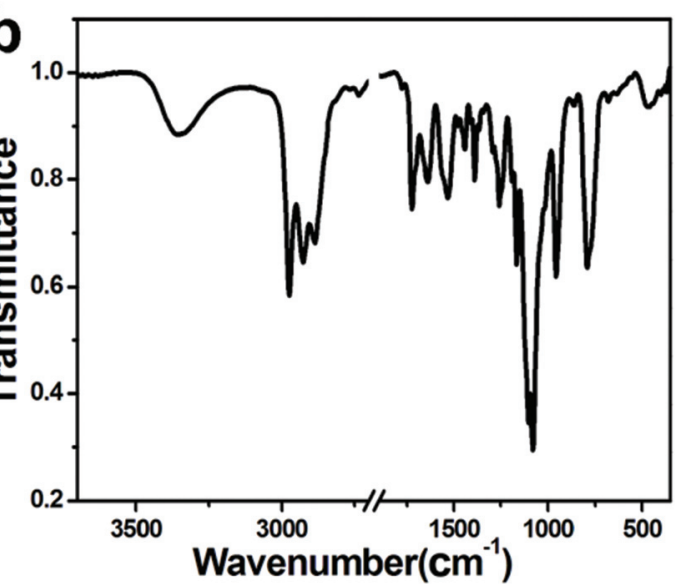

Fig. 4 (a) MSN-4 functionalized with Schiff based-TPA and (b) FTIR spectra of MSN-4 functionalized with Schiff based-TPA.

In our synthesis process of mixed anionic surfactant-templated mesoporous materials, APTS worked as the CSDA agent to form mesopores and tune the nanoparticle morphology with a grafting moiety for post-grafting.

Schiff based TPA-functionalized MSN-4 featured excellent water dispersity and displayed blue fluorescence at $428 \mathrm{~nm}$ in water. With $2 \mathrm{ppm}$ of $\mathrm{Fe}^{3+}$ added each time, the fluorescence intensity of Schiff based TPA-functionalized MSN-4 was gradually quenched, which could be ascribed to a PET (photoinduced energy transfer) mechanism and/or a paramagnetic quenching effect of $\mathrm{Fe}^{3+} .^{48}$ Thus, Schiff based TPA-functionalized MSN-4 could be used as a fluorescence sensing material to detect $\mathrm{Fe}^{3+}$ in water.

We also tested the selective detection ability of Schiff based TPA-functionalized MSN-4 to other ions such as $\mathrm{Fe}^{2+}, \mathrm{Cu}^{2+}$, $\mathrm{Na}^{+}, \mathrm{Zn}^{2+}, \mathrm{Cr}^{3+}, \mathrm{Co}^{2+}, \mathrm{Ni}^{2+}, \mathrm{Mn}^{2+}$ and $\mathrm{Mg}^{2+}$, as shown in Fig. 5b. No significant shape change or intensity decrease was observed during the addition of the other ions in comparison with the addition of equivalent $\mathrm{Fe}^{3+}$ (the detailed experimental conditions for $\mathrm{Fe}^{3+}$ sensing and the detection selectivity are given in the ESI $\dot{\dagger}$ ). This result may be attributed to the fact that no PET of the quenching process occurred by other ion influences. Thus, selective $\mathrm{Fe}^{3+}$ detection materials have been successfully synthesized by introducing the Schiff base TPA onto the surface of MSN-4 by using a conjugated bond.
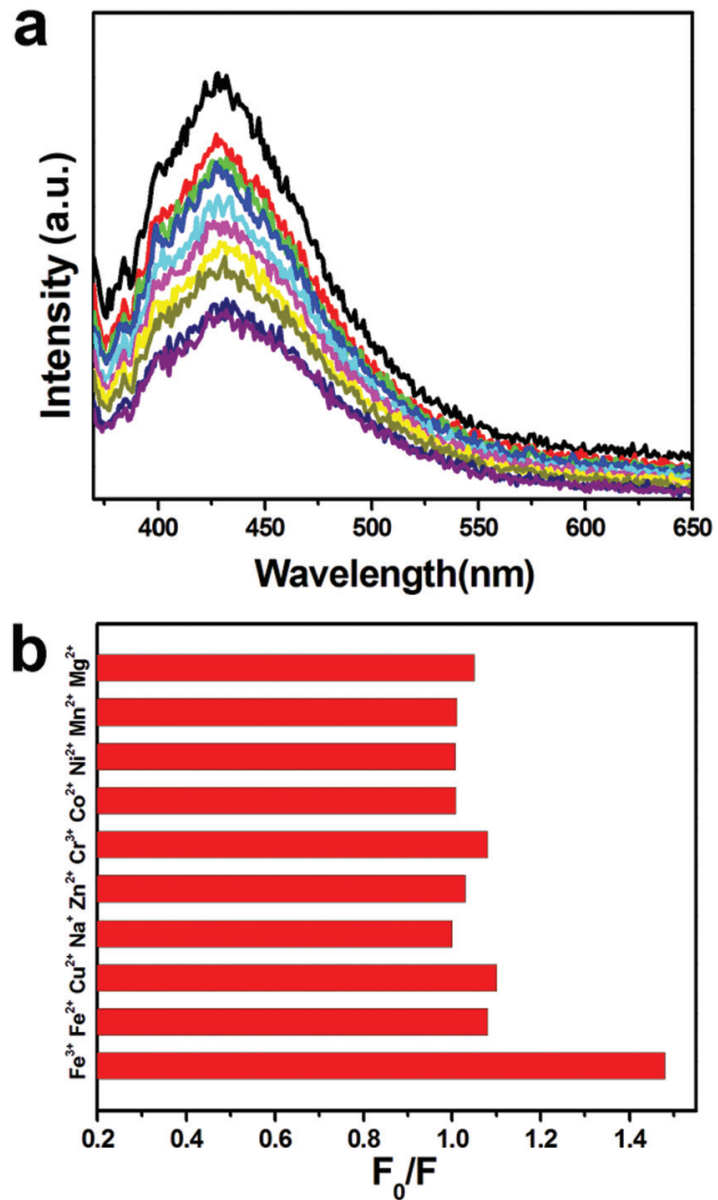

Fig. 5 (a) Fluorescence emission spectra of Schiff based TPA-functionalized $\mathrm{MSN}-4$ performed by adding an increasing amount of $\mathrm{Fe}^{3+}$ (excited at $350 \mathrm{~nm}$ ) and (b) selectivity of $\mathrm{Fe}^{3+}$.

\section{Conclusions}

In summary, this work demonstrated the synthesis of large pore mesoporous silica nanoparticles with a uniform size by using a mixed anionic surfactant as the template without a polymer micelle or swelling agent. The dispersity, mesopore structure and the morphology of mesoporous silica nanoparticles could be tailored by adjusting the concentration of anionic surfactants and the co-structure-directing agent. The formation process of MSNs was nucleation and growth. A post-grafting method has been used to introduce TPA on the surface of nanoparticles, which performed good selectivity detection of $\mathrm{Fe}^{3+}$ in aqueous media.

\section{Acknowledgements}

The authors would like to thank Professor Shunai Che for her valuable suggestions about mechanism. Dr Fangyuan Gai is grateful for Kempestiftelserna for postdoctoral fellowship. Dr Fangyuan Gai is grateful to Professor Otto S. Wolfbeis for his encouragement. This work was supported by the National Natural Science Foundation of China (no. 21501018 and 51503190). 


\section{Notes and references}

1 Y. Chen, H. R. Chen, S. J. Zhang, F. Chen, L. X. Zhang, J. M. Zhang, M. Zhu, H. X. Wu, L. M. Guo, J. W. Feng and J. L. Shi, Adv. Funct. Mater., 2011, 21, 270-278.

2 M. Wu, Q. Meng, Y. Chen, Y. Du, L. Zhang, Y. Li, L. Zhang and J. Shi, Adv. Mater., 2015, 27, 215-222.

3 J. Wei, Y. Y. Liu, J. Chen, Y. H. Li, Q. Yue, G. X. Pan, Y. L. Yu, Y. H. Deng and D. Y. Zhao, Adv. Mater., 2014, 26, 1782-1787.

4 Y. Deng, C. Liu, T. Yu, F. Liu, F. Zhang, Y. Wan, L. Zhang, C. Wang, B. Tu, P. A. Webley, H. Wang and D. Zhao, Chem. Mater., 2007, 19, 3271-3277.

5 F. Tang, L. Li and D. Chen, Adv. Mater., 2012, 24, 15041534.

6 J. Li, Q. Hu, H. Tian, C. Ma, L. Li, J. Cheng, Z. Hao and S. Qiao, J. Colloid Interface Sci., 2009, 339, 160-167.

7 S. H. Wu, C. Y. Mou and H. P. Lin, Chem. Soc. Rev., 2013, 42, 3862-3875.

8 D. K. Shen, J. P. Yang, X. M. Li, L. Zhou, R. Y. Zhang, W. Li, L. Chen, R. Wang, F. Zhang and D. Y. Zhao, Nano Lett., 2014, 14, 923-932.

9 S.-H. Chiou, S.-F. Jang and C.-Y. Mou, Nanomedicine, 2014, 9, 377-380.

10 K.-C. Kao, T.-S. Lin and C.-Y. Mou, J. Phys. Chem. C, 2014, 118, 6734-6743.

11 K.-C. Kao and C.-Y. Mou, Microporous Mesoporous Mater., 2013, 169, 7-15.

12 S. Shen, T. Gu, D. Mao, X. Xiao, P. Yuan, M. Yu, L. Xia, Q. Ji, L. Meng, W. Song, C. Yu and G. Lu, Chem. Mater., 2012, 24, 230-235.

13 Z. Huang and S. Che, Bull. Chem. Soc. Jpn., 2015, 88, 617632.

14 L. Han and S. Che, Chem. Soc. Rev., 2013, 42, 3740-3752.

15 C. Gao and S. Che, Adv. Funct. Mater., 2010, 20, 2750-2768.

16 H. Qiu and S. Che, Chem. Soc. Rev., 2011, 40, 1259-1268.

17 X. Wu, H. Qiu and S. Che, Microporous Mesoporous Mater., 2009, 120, 294-303.

18 B. Li, Y. Pu, Y. Li and Y. Yang, Mater. Lett., 2013, 113, 3437.

19 Y. Li, B. Li, Z. Yan, Z. Xiao, Z. Huang, K. Hu, S. Wang and Y. Yang, Chem. Mater., 2013, 25, 307-312.

20 L.-L. Li, H. Sun, C.-J. Fang, Q. Yuan, L.-D. Sun and C.-H. Yan, Chem. Mater., 2009, 21, 4589-4597.

21 D. Niu, Z. Ma, Y. Li and J. Shi, J. Am. Chem. Soc., 2010, 132, 15144-15147.

22 Y. Zhao, L. Zhao, G. Wang and Y. Han, Chem. Mater., 2011, 23, 5250-5255.

23 Y. Han, L. Zhao and J. Y. Ying, Adv. Mater., 2007, 19, 24542459.

24 Y. Han, D. Zhang, L. L. Chng, J. Sun, L. Zhao, X. Zou and J. Y. Ying, Nat. Chem., 2009, 1, 123-127.
25 H. M. Nan, Q. Cai, Q. Y. Sun, J. J. Qi, Y. S. Liu, H. Zhou, Y. X. Yu, W. L. Shi, T. S. Wang, C. M. Xu and H. D. Li, Microporous Mesoporous Mater., 2011, 142, 585-591.

26 Q. Huo, J. Liu, L.-Q. Wang, Y. Jiang, T. N. Lambert and E. Fang, J. Am. Chem. Soc., 2006, 128, 6447-6453.

27 N. Z. Knezevic and V. S. Y. Lin, Nanoscale, 2013, 5, 15441551.

28 J. Wei, Y. H. Li, M. H. Wang, Q. Yue, Z. K. Sun, C. Wang, Y. J. Zhao, Y. H. Deng and D. Y. Zhao, J. Mater. Chem. A, 2013, 1, 8819-8827.

29 H.-Y. Hsueh, C.-T. Yao and R.-M. Ho, Chem. Soc. Rev., 2015, 44, 1974-2018.

30 F. Gai, T. Zhou, Y. Liu and Q. Huo, J. Mater. Chem. A, 2015, 3, 2120-2127.

31 N. Z. Knezevic and J.-O. Durand, Nanoscale, 2015, 7, 21992209.

32 F. Chi, Y.-N. Guo, J. Liu, Y. Liu and Q. Huo, J. Phys. Chem. C, 2010, 114, 2519-2523.

33 Y. F. Shi, B. Li, P. Wang, R. Dua and D. Y. Zhao, Microporous Mesoporous Mater., 2012, 155, 252-257.

34 X. Zhou, S. Qiao, N. Hao, X. Wang, C. Yu, L. Wang, D. Zhao and G. Q. Lu, Chem. Mater., 2007, 19, 1870-1876.

35 J. Peng, J. Liu, J. Liu, Y. Yang, C. Li and Q. Yang, J. Mater. Chem. A, 2014, 2, 8118-8125.

36 S. Y. Bai, J. Liu, J. S. Gao, Q. H. Yang and C. Li, Microporous Mesoporous Mater., 2012, 151, 474-480.

37 J. Liu, S. Bai, H. Zhong, C. Li and Q. Yang, J. Phys. Chem. C, 2010, 114, 953-961.

38 Y. Shi, B. Li, P. Wang, R. Dua and D. Zhao, Microporous Mesoporous Mater., 2012, 155, 252-257.

39 Q. Wu, F. Zhang, J. Yang, Q. Li, B. Tu and D. Zhao, Microporous Mesoporous Mater., 2011, 143, 406-412.

40 N. Hao, H. Wang, P. A. Webley and D. Zhao, Microporous Mesoporous Mater., 2010, 132, 543-551.

41 J. Wang, Q. Xiao, H. Zhou, P. Sun, Z. Yuan, B. Li, D. Ding, A.-C. Shi and T. Chen, Adv. Mater., 2006, 18, 3284-3288.

42 K. Zhang, L.-L. Xu, J.-G. Jiang, N. Calin, K.-F. Lam, S.-J. Zhang, H.-H. Wu, G.-D. Wu, B. Albela, L. Bonneviot and P. Wu, J. Am. Chem. Soc., 2013, 135, 2427-2430.

43 L. Xiong, X. Du, B. Shi, J. Bi, F. Kleitz and S. Z. Qiao, J. Mater. Chem. B, 2015, 3, 1712-1721.

44 L. Han, Q. Chen, Y. Wang, C. Gao and S. Che, Microporous Mesoporous Mater., 2011, 139, 94-103.

45 J. M. Rosenholm, J. Zhang, W. Sun and H. Gu, Microporous Mesoporous Mater., 2011, 145, 14-20.

46 Z. Sun, Q. Yue, Y. Liu, J. Wei, B. Li, S. Kaliaguine, Y. Deng, Z. Wu and D. Zhao, J. Mater. Chem. A, 2014, 2, 1832218328.

47 D. Niu, Z. Liu, Y. Li, X. Luo, J. Zhang, J. Gong and J. Shi, Adv. Mater., 2014, 26, 4947-4953.

48 Q. Meng, W. Su, C. He and C. Duan, Talanta, 2012, 97, 456-461. 